\title{
INTERMOLECULAR ASSOCIATION OF AMPHIPATHIC POLYELECTROLYTES IN AQUEOUS SOLUTIONS
}

\author{
HERNÁN E. RÍOS*, MARCELA D. URZÚA, VALERIA VILLALOBOS AND VÍCTOR A. VARGAS \\ Departamento de Química. Facultad de Ciencias. Universidad de Chile. \\ Casilla 653. Correo Central. Santiago. CHILE
}

\begin{abstract}
The intermolecular association of amphipathic polyelectrolytes is studied using fluorescence and conductivity methods. This type of interaction is evidenced by the decrease in the average distances between charges as the polyelectrolyte side chain increases in length. This modify the dissociation degree and consequently, the linear charge density parameter. These distances were calculated with the Manning counterion condensation theory for the conductivity of polyelectrolyte solutions. The determination of the ratio $\mathrm{I}_{1} / \mathrm{I}_{3}$ of the fluorescence bands of pyrene with polymer content reveals the formation of hydrophobic microdomains at very low concentrations, smaller than the required concentration to produce a significant change in the average distances between charges. These distances and also the $\mathrm{I}_{1} / \mathrm{I}_{3}$ ratio vary with polymer concentration and their values were dependent on the size of the side chain in the polyelectrolyte. Finally, the experimental behavior of the solution viscosity and electrical conductivity of polyelectrolytes, which increase drastically with dilution, can be explained as a continuous change in the average distances between charges which produce conformational changes.
\end{abstract}

\section{INTRODUCTION}

From years the conductivity properties of polyelectrolytes have been used as a tool for detect conformational changes in this type of macromolecules in aqueous solution. In fact, changes in the average distance between charges, $\mathbf{b}$, on the polyion has been used as a criterion to account for these conformational changes. In fact, Manning`s counterion condensation theory allows to determine $\mathbf{b}$ by electric conductivity measurements. ${ }^{1-4}$ This theory relates the average distances between charges on the polyelectrolyte, $\mathbf{b}$, framework with the linear charge density parameter, $\xi$, through the following equation ${ }^{2}$ :

$$
\xi=\frac{e^{2}}{\varepsilon k_{b} T b}
$$

Where, e is the elementary charge, $\varepsilon$ the solvent dielectric constant, $k_{b}$ the Boltzmann constant and T the absolute temperature. Parameters $\xi$ and $\mathbf{b}$ are can be determined experimentally through specific electrical conductivity measurements of polyelectrolyte in aqueous solutions, $\kappa$, from which it is possible to determine the molar conductivity, $\Lambda$. The limiting law for the electrical conductivity of a polyelectrolyte solution is related to $\xi$ through the following equation:

$$
\Lambda=f\left(\lambda_{i}^{\circ}+\lambda_{\mathrm{p}}\right)
$$

Where $\lambda_{i}^{\circ}$ and $\lambda_{\mathrm{p}}$ are the counterion and the polyion limiting molar conductivities, respectively and $f$ is a polyelectrolyte-counterion interaction parameter related to $\xi$ through:

$$
f=0.866 \xi^{-1}
$$

The determination of $f$ is not always possible, thus a relative interaction parameter, $f^{*}$, referred to the value that the polyion should have in the completely extended conformation, has been proposed. ${ }^{3}$

$$
f^{*}=\frac{\Lambda_{\text {exp }}^{0}}{\Lambda_{p, \text { theor }}^{0}}
$$

The calculation of $f^{*}$ requires the determination of $\Lambda_{\text {exp }}^{0}$, the experimental limiting molar conductivity, and assuming that $\Lambda_{p, \text { theor }}^{\circ}$ is correctly given by
Manning's theory. ${ }^{1}$

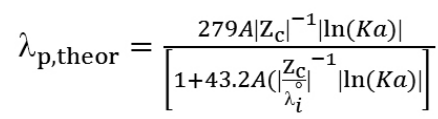

Here,

$$
A=\frac{4 \varepsilon_{0} \varepsilon_{r} k_{b} T}{3 \eta e}
$$

and,

$$
K^{2}=\left(\frac{e^{2}}{\varepsilon_{0} \varepsilon_{r} k_{b} T}\right) \times \xi^{-1} n_{s}
$$

In equations 4,5 and $6, \boldsymbol{a}$ is the polyion radius, $\mathrm{Z}_{\mathrm{c}}$, the counterion charge, $\eta$, the absolute viscosity of the solvent and $n_{s}$ the stoichiometric concentration of monomer units in mol $\times \mathrm{L}^{-1}$, respectively. Thus to evaluate conformational changes in some works, the determination of $\mathbf{b}$ values from conductivity measurements have been determined as a function of $\mathrm{Cp}$ and also as a function of the solution temperature

This procedure was applied by Izumrudov in order to study the conformation of several symmetric ionene bromides in salt-free aqueous solutions. ${ }^{5}$ The obtained $\mathbf{b}$ values were considered as a characteristic measurement of the conformation that these polyelectrolytes adopt in solution. For instance, the decrease in the degree of polymerization (DP) for a 3,3-ionene produces an increase in $\mathbf{b}$, suggesting a transition from coil-like to rod-like conformation. On the other hand, Davydova and Izumrudov reported the conformation of vinylic polyelectrolytes, such as poly(acrylate), poly(styrene sulfonates) and rigid native DNA, as a function of temperature. ${ }^{6}$ The trend of $\mathbf{b}$ values depends on the studied system. In fact, for synthetic polyelectrolytes, $\mathbf{b}$ increases monotonically with temperature whereas for DNA an abrupt change in $\mathbf{b}$ values is observed. For the vinyl-type polyanions, a conformational transition was observed in the same DP region, $10^{2}$ to $10^{3}$, whereas for native DNA, macromolecules undergo a conformational change at higher DP values, $10^{3}-10^{4}$, at nearly the same range of temperature between $50^{\circ} \mathrm{C}$ to $65^{\circ} \mathrm{C}$. The influence of the alkyl side chain on exhaustively alkylated poly( $\mathrm{N}$-alkyl-4-vinylpyridinium) bromides with alkyl = ethyl, propyl and butyl has been also investigated. ${ }^{7}$ The main findings for polyelectrolytes with alkyl side chain at a constant DP of 100 were: $\mathbf{b}$ values decreases as the side chain length increases and practically no effect of the temperature on the $\mathbf{b}$ values was observed, remaining practically constant from 20 to $70^{\circ} \mathrm{C}$. Side chain chains with hydrophilic hydroxyethyl and hydroxypropyl groups showed larger $\mathbf{b}$ values than their hydrophobic analogs suggesting a more extended conformation in these cases, probably from a coillike to a rod-like conformation due to their higher hydrophilic character.

Poly(N-ethylpyridinium) bromide and asymmetric $(2, n)$ ionene bromides were also studied, particularly, $(2,4),(2,8)$ and $(2,10)$ ionenes. ${ }^{8}$ The results revealed that conformational changes of these chains are dependent on the variation of charge density, no matter if the charges on the pyridinium polyelectrolyte are randomly distributed along the chains or, in the case of ionenes, are regularly spaced in their chains.

In all these works, the attached alkyl side chains were very short, from methyl to butyl. Practically in the literature there are no works considering cationic polyelectrolytes containing larger side chains.

In a recent work, we found that the intrinsic viscosities of aqueous solutions of poly (n-alkylmethyldiallyl ammonium) chlorides with n-alkyl $=$ octyl, decyl and dodecyl drastically decreases when compared to that of poly(diallyldimethyl ammonium) chloride, PDDMAC, the hydrophilic precursor from where these polyelectrolytes are obtained. ${ }^{9}$ The results are interpreted as a hypercoiling process of the alkylated derivatives due to the formation of hydrophobic microdomains produced by the interaction among 
non-polar aliphatic side chains. Unpublished results of electrical conductivity of their aqueous solutions suggest that the dissociation degree of these polyelectrolytes decreases when their side chains increases. Thus, if the last is correct, the average distances between charges should be changing accordingly.

Therefore, in the present work b values for cationic polyelectrolytes containing large side chains are calculated from electrical conductivity measurements. The main objective is to establish if there is a dependence of $\mathbf{b}$ on the polyelectrolyte concentration, consistent with a conformational change as it can be assumed by the solution viscosity behavior of these macromolecules. ${ }^{9}$ The $\mathbf{b}$ values here found are correlated with the behavior of the ratio $\left(I_{1} / I_{3}\right)$ of the fluorescence vibronic bands of pyrene, which have been widely used to evidence hydrophobic microdomains.

\section{MATERIALS AND METHODS}

The used polyelectrolytes were a series of series of poly (n-alkylmethyldiallylammonium) chloride generically named $\mathrm{PCn}$, with $\mathrm{n}$-alkyl $=8,10$ and 12 . Poly(N,N-dimethyl-N,N-diallylammonium) chloride, the most hydrophilic polyelectrolyte was chosen as a reference to compare the conductivity properties of the amphipathic polyelectrolytes corresponding to $\mathrm{PCn}$ with $\mathrm{n}=1$. The structure can be observed in the following scheme:
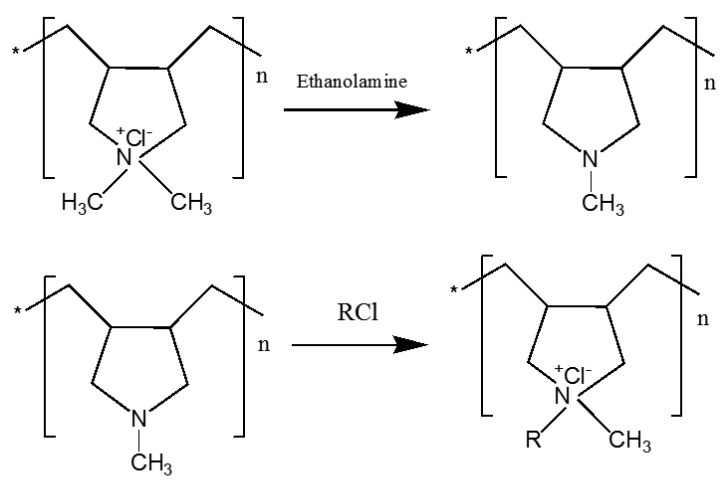

$\mathrm{PC}$

PCn

The synthesis details have been described elsewhere..$^{10,11}$

Conductivity measurements were performed at $25 \pm 0.1^{\circ} \mathrm{C}$ under nitrogen atmosphere using a CDM-85 Radiometer Research Conductimeter with a conductivity cell of bright platinum electrodes with $1.295 \mathrm{~cm}^{-1}$ cell constant. All conductivity measurements were highly reproducible and it were performed at least three times. De-ionized water with a specific conductivity between 0.5 and $0.7 \mu \mathrm{S} \times \mathrm{cm}^{-1}$ was used for the solutions. The polyelectrolyte concentration in moles monomer units $\mathrm{L}^{-1}$ was named $\mathrm{Cp}$. The molecular weight of $\mathrm{PCn}$ was in the 10,000 to 30,000 range.

The average distance among charges, $\mathbf{b}$, was determined by adjusting the experimental data for the specific conductivity of the polyelectrolyte solution to the following equation:

$$
\kappa\left(C_{p}\right)-\left(\kappa^{0}+f^{*} \times 10^{-3} \times L_{b}^{-1} \times b \times C_{p}\right) \times\left(\lambda_{c}^{0}+\frac{279 \times A \times|Z c|^{-1} \times|L n K a|}{1+43.2 \times A \times\left(Z c \times \lambda_{c}^{0}\right)^{-1} \times|L n K a|}\right)=0
$$

Where $L_{b}$ is the Bjerrum length $7.1429 * 10^{-8} \mathrm{~cm}$. Thus, according to equation $6, K$ can be calculated as:

$$
K=2.99601 \times 10^{-5}\left(7.1429 \times 10^{-8} b \times C_{p}\right)^{1 / 2}
$$
7 is:

Considering that the polyion radius $a$ is $1.5 \times 10^{-8} \mathrm{~cm}$, then $K a$ in equation

$$
K a=2.99601 \times 10^{-5} \times 1.5 \times 10^{-8}\left(7.1429 \times 10^{-8} b \times C_{p}\right)^{1 / 2}
$$

Finally, replacing all this values in equation 7 , the following expression can be obtained:

$$
\kappa\left(C_{p}\right)-\left(\kappa^{0}+f^{*} \times 10^{-3} \times L_{b}^{-1} \times b \times C_{p}\right) \times
$$

$\times\left(76.35+\frac{22.339 \times\left|\operatorname{Ln}\left(2.996 \times 10^{-5}\right) \times 1.5 \times 10^{-8} \times\left(b \times C_{p}\right)^{1 / 2}\right|}{1+4.941 \times 10^{-2} \times(76.35)^{-1} \times\left|\operatorname{Ln}\left(2.996 \times 10^{-5}\right) \times 1.5 \times 10^{-8} \times\left(b \times C_{p}\right)^{1 / 2}\right|}\right)=0$

The latter equation can be written as $\kappa\left(C_{p}\right)-\kappa_{\text {Manning }}=0$. Thus, for each measured $\kappa$ as a function of $C p$, $\mathbf{b}$ can be determined calculating the $\mathbf{b}$ values at which $\kappa\left(C_{p}\right)-\kappa_{\text {Manning }}=0$. equation 11 becomes zero.

The relation between the fluorescence intensity vibronic bands of pyrene $\mathrm{I}_{1} / \mathrm{I}_{3}$ was determined from the fluorescence spectra registered in the range 350 $\mathrm{nm}$ to $420 \mathrm{~nm}$, using an excitation wave length of $330 \mathrm{~nm}$ with a spectral band width of $1.0 \mathrm{~nm}$ in the emission monocromator. The pyrene concentration was $2.0 \mu \mathrm{M}$.

\section{RESULTS AND DISCUSSION}

Values of $\kappa$ from the polyelectrolyte solutions are shown in Figure 1. As can be seen there is a smooth increase in $\kappa$ with $\mathrm{Cp}$. However, in all cases, increasing $\mathrm{Cp}$, curves bend downward at apparently the same $\mathrm{Cp}$ range, at least for the PCn polyelectrolytes containing large side chains. This effect is less marked in the case of PC1 where the curve appears to increase linearly as $\mathrm{Cp}$ increases. Changes in specific conductivity can be attributed to the effect of higher order ionic interactions on electrical conductivity as $\mathrm{Cp}$ increases. ${ }^{12}$

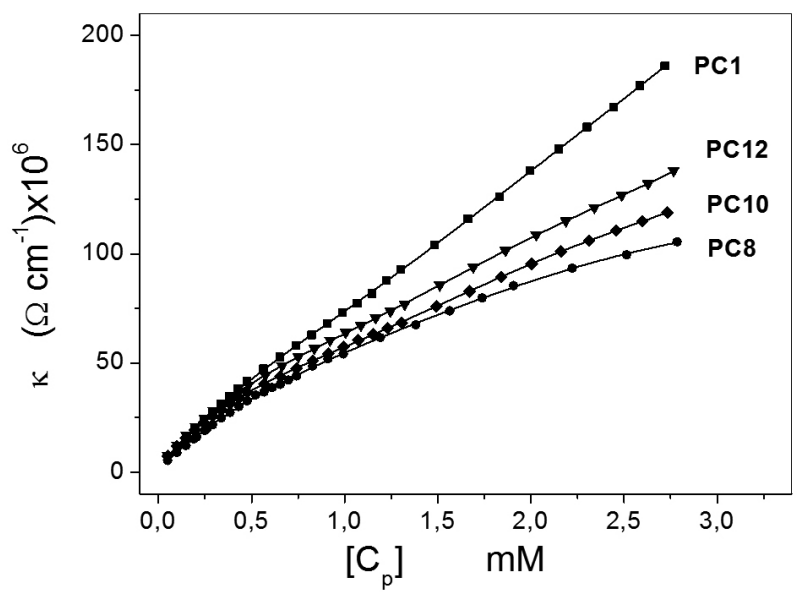

Fig. 1:Specific conductivity behavior of polyelectrolyte solutions with $\mathrm{Cp}$.

In Figure 2 Kohlrausch plots are displayed. As can be seen, $\Lambda$ increases exponentially with dilution in all cases corresponding to a weak electrolyte behavior. The $\Lambda$ trend is also dependent on the polyelectrolyte side chain length following the order: PC12 > PC10 > PC8. The more hydrophilic polyelectrolyte, $\mathrm{PC}$, shows a slightly different behavior as compared with PCn with large side chains. The PC1 trend it compares well with PC8 behavior but at greater $\Lambda$ values.

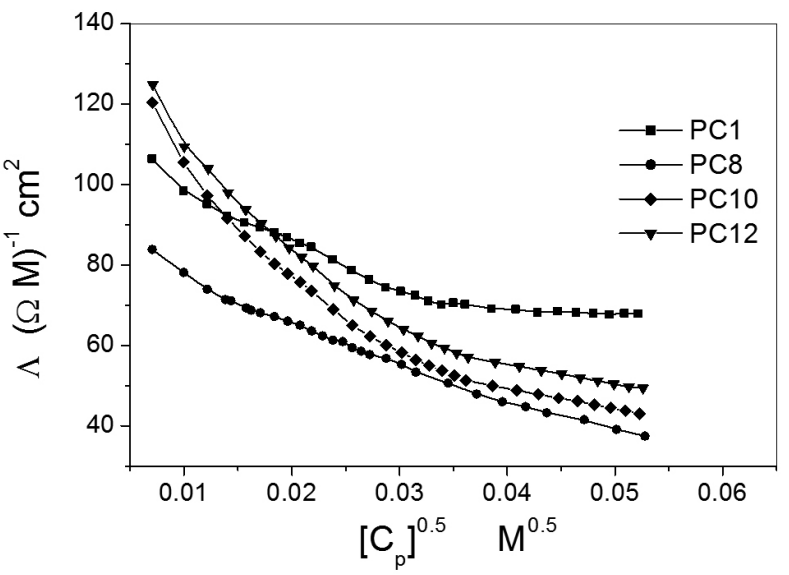

Fig. 2: Kohlrausch plots for polyelectrolyte solutions. 
The analysis here proposed requires the determination of, the limiting equivalent conductivity, which is not possible to determine with some accuracy from the plots of figure 2. Therefore, the well known analysis of Krauss- Bray was used to determine. In figure 3, the experimental data are plotted according to the Krauss-Bray linear equation:

$$
\frac{1}{\Lambda}=\frac{1}{\Lambda^{0}}+\frac{C p \times \Lambda}{K_{d i s s} \times \Lambda^{2}}
$$

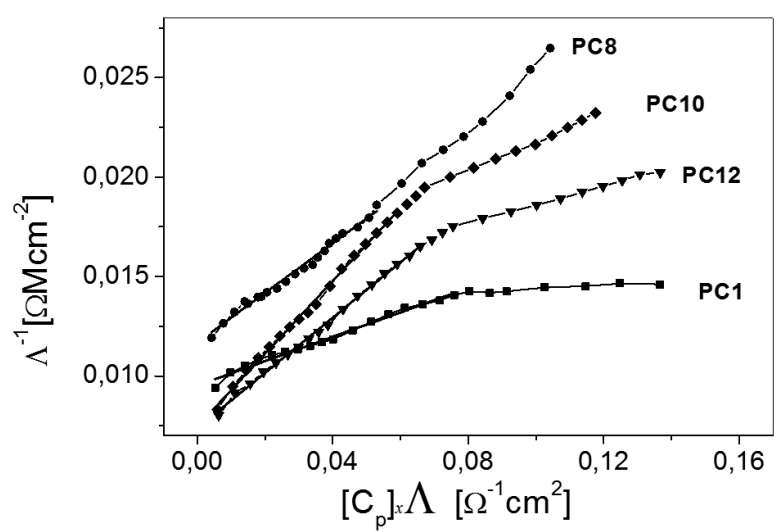

Fig. 3: Krauss-Bray plots for polyelectrolyte solutions.

As can be seen in Figure 3, at low values of all the curves behave almost linearly, thus the extrapolation from this linear region to $\operatorname{Cp} \times \Lambda=0$, allows to determine $\Lambda_{\text {exp }}^{0}$ and an unique value of $\mathrm{K}_{\text {diss }}$ from the slope. The values of here found, although smaller than that reported by Barraza et al., for dicarboxylate-type polyelectrolytes with large aliphatic side chains, show the same dependence with the size of the polyelectrolyte side chain, i.e., the larger the polyelectrolyte side chain, the smaller $\Lambda_{\text {exp }}^{0}{ }^{13}$ The values of $\Lambda_{\text {exp }}^{0}$ together with the magnitudes of $f^{*}$, calculated according equation 4 , are summarized in Table 1. Both $\Lambda_{\exp }^{0}$ values and $\mathrm{K}_{\text {diss }}$ values are certainly dependent on the kind of polyelectrolyte. The $\Lambda_{\text {exp }}^{0}$ values were determined with some confidence but the unique value of $\mathrm{K}_{\text {diss }}$ obtained is uncertain because the dissociation degree $\alpha$ depends on the polyelectrolyte linear charge density $\xi$, and the latter on Cp. Thus in the case of polyelectrolytes an apparent $\mathrm{K}_{\text {diss }}$ is generally reported. However, the values of $\mathrm{K}_{\text {diss }}$ here found correspond to weak electrolyte.

Table 1: Limiting molar conductivity, $\mathrm{K}_{\mathrm{dis}}$, and $f^{*}$ for polyelectrolytes

\begin{tabular}{|c|c|c|c|}
\hline $\mathrm{PCn}$ & $\Lambda^{\mathrm{o}}(\Omega \times \mathrm{M})^{-1} \mathrm{~cm}^{2}$ & $\mathrm{~K}_{\text {diss }}$ & $f^{*}$ \\
\hline $\mathrm{PC} 1$ & $105.1 \pm 1.3$ & $1.48 \mathrm{E}-3 \pm 0.10 \mathrm{E}-3$ & 0.278 \\
\hline $\mathrm{PC} 8$ & $85.6 \pm 1.1$ & $1.09 \mathrm{E}-3 \pm 0.07 \mathrm{E}-3$ & 0.226 \\
\hline PC10 & $128.2 \pm 2.3$ & $0.35 \mathrm{E}-3 \pm 0.02 \mathrm{E}-3$ & 0.341 \\
\hline PC12 & $138.1 \pm 2.3$ & $0.37 \mathrm{E}-3 \pm 0.02 \mathrm{E}-3$ & 0.366 \\
\hline
\end{tabular}

A plot showing how the dissociation degree depends on $\mathrm{Cp}$ is displayed in figure 4 . The values of $\alpha$ calculated as the quotient, $\Lambda(\mathrm{Cp})$, is dependent

$$
\overline{\Lambda_{\exp }^{0}}
$$

on the side chain length following the sequence: $\mathrm{PC} 1>\mathrm{PC} 8>\mathrm{PC} 12 \cong \mathrm{PC} 0$. Thus, the behavior of $\alpha$ suggests that the average linear charge distances decreases as $\mathrm{Cp}$ increases. Consequently, the intermolecular association between side chains as $\mathrm{Cp}$ increases appears to be a plausible explanation for this phenomenon.

In order to elucidate this possibility, the ratio $\mathrm{I}_{1} / \mathrm{I}_{3}$ of the fluorescence vibronic bands of pyrene was measured as a function of $\mathrm{Cp}$. These results are shown in Figure 5. Abrupt changes in the magnitude of this ratio at different ranges of concentration can be observed, suggesting that the polyelectrolyte are actually forming hydrophobic micro domains, probably by interaction among inter polymer side chains, at least in the case of the polyelectrolytes containing large aliphatic side chains in their structure.

The $\mathrm{Cp}$ at which this phenomenon occurs, the inflection point in curves of
Figure 5, is known as critical aggregation concentration CAC. In the present case the values of the CAC were $3.4 \times 10^{-5} \mathrm{M}, 8.5 \times 10^{-4}$ and $4.2 \times 10^{-3} \mathrm{M}$ for PC12, PC10 PC8, respectively. Thus, the larger the polyelectrolyte side chain, the smaller the CAC. According to these results, PC1 is unable to form these hydrophobic micro domains in the concentration range here studied. Therefore, if the polyelectrolytes are shrinking by hydrophobic interactions, their average length between charges should be changing with $\mathrm{Cp}$. Consequently, the $\mathbf{b}$ values were calculated according to equation 11 and the obtained results are summarized in Table 2 and plotted in Figure 6. As can be observed, at constant $\mathrm{Cp}$, b values decrease as the polyelectrolyte side chain length increase. On the other hand, for the same polyelectrolyte, $\mathbf{b}$ values decrease as $\mathrm{Cp}$ increase.

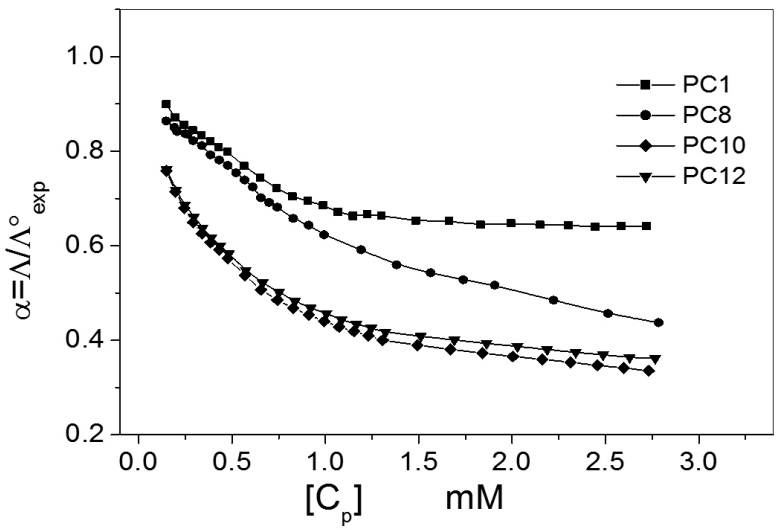

Fig. 4: Dissociation degree of polyelectrolytes versus $\mathrm{Cp}$.

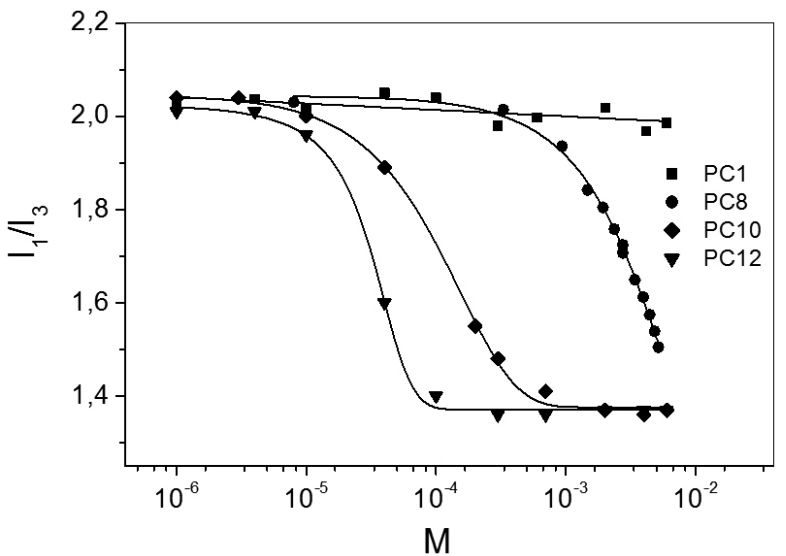

Fig. 5: $\mathrm{I}_{1} / \mathrm{I}_{3}$ ratio of fluorescence bands of pyrene versus $\mathrm{Cp}$.

Table 2: Values of the average distances between charges.

\begin{tabular}{|c|c|c|c|c|}
\hline $\begin{array}{c}\text { Concentration } \\
(\mathrm{mM})\end{array}$ & $\begin{array}{c}\mathrm{PC}_{1} \\
\boldsymbol{b}, \AA \\
f^{*}=(0.278)\end{array}$ & $\begin{array}{c}\mathrm{PC}_{8} \\
\boldsymbol{b}, \AA\end{array}$ & $\begin{array}{c}\mathrm{PC}_{10} \\
\boldsymbol{b}, \AA \\
f^{*}=(0.226)\end{array}$ & $\begin{array}{c}\mathrm{PC}_{12} \\
\boldsymbol{b}, \AA \\
f^{*} \\
=(0.341)\end{array}$ \\
\hline 0.05 & 7.65 & 7.10 & 6.74 & 6.53 \\
\hline 0.20 & 6.45 & 6.19 & 5.18 & 5.15 \\
\hline 0.30 & 6.26 & 6.01 & 4.71 & 4.76 \\
\hline 0.48 & 5.91 & 5.64 & 4.17 & 4.20 \\
\hline 1.50 & 4.82 & 4.00 & 2.84 & 2.95 \\
\hline 2.00 & 4.82 & 3.79 & 2.68 & 2.80 \\
\hline 2.75 & 4.77 & 3.22 & 2.46 & 2.62 \\
\hline
\end{tabular}

This behavior is consistent with a compact coil conformation of the polyelectrolyte as $\mathrm{Cp}$ increases, which is also coherent with the trend followed by the intrinsic viscosity of their aqueous solutions. ${ }^{10}$ As expected, in the case of $\mathrm{PC} 1$, the decrease in $\mathbf{b}$ values is less pronounced, consistent with their 
greater hydrophilic character, and because in this case, side chains capable to interact each other are absent. For nearly the same range of concentration, the b values here found decreased in a wider range of distances than that reported by Izumrudov for 3,3-, 4,4-, 6,6,- and 10,10- ionenes (5). This effect may be attributed to the different type of polyelectrolyte. In the present case, the side aliphatic chains are probably move more freely to interact among them. This possibility may be less favorable in linear ionenes, where the aliphatic methylene groups are less free to interact because they are linking neighboring ammonium groups.

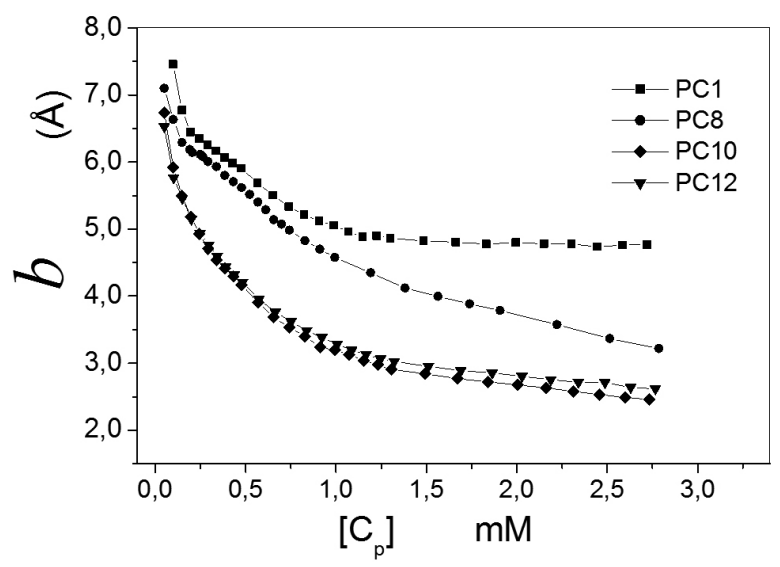

Fig. 6: Average distances between charges as a function of $\mathrm{Cp}$

\section{CONCLUSIONS}

From the above results it can be concluded that conductivity measurement of polyelectrolyte solution allows determining the average distances between charges in a polyion, therefore their linear charge density. As it is well known, viscosity and electrical conductivities of polyelectrolyte solutions, drastically increases with dilution. This phenomenon has been attributed to an increase in the polyelectrolyte dissociation degree producing a consequent increase in their hydrodynamic volume by electrostatic repulsion between charges on the polyion. In the present case, the amphipathic polyelectrolytes associate themselves by intermolecular hydrophobic effect between aliphatic side chains, which bring about a change in $\mathbf{b}$ and therefore in the dissociation degree. In the same range of $\mathrm{Cp}$, the changes in $\mathbf{b}$ here found are larger than those reported by Izumrudov for ionenes where probably these hydrophobic interactions are more hindered. ${ }^{5}$ The behavior of the ratio $\mathrm{I}_{1} / \mathrm{I}_{3}$ of the fluorescence vibronic bands of pyrene reveals that the formation of hydrophobic micro domains starts at a very small $\mathrm{Cp}$, a concentration which is lower than that required to produce a significant change in $\mathbf{b}$ values as those detected by the conductivity method. Manning's theory overestimates the values of conductivity because these polyelectrolytes have a non-linear conformation proposed by the theory. However, if the latter is used as a reference state, it is possible to explain the empirical behavior of the electrical conductivity of polyelectrolyte solutions.

\section{ACKNOWLEDGEMENTS}

The Program PEEI from the Universidad de Chile is grateful acknowledged.

\section{REFERENCES}

1. G. Manning, J. Phys. Chem. 79, 262, (1975)

2. G. Manning, J. Chem. Phys. 51, 924, (1969)

3. H. Ríos, R. Barraza, I. Gamboa. Polym. Int. 31, 213, (1993)

4. H. Ríos. Polym. Int. 50, 885, (1993).

5. A. Zelikin, O. Davydova, N. Akritskaya, S. Kargov, V. Izumrudov. J. Phys.Chem. 108, 490, (2004)

6. O. Davydova, A. Zelikin, S. Kargov, V. Izumrudov. Macromol. Chem. Phys., 202, 1361, (2001).

7. O. Davydova, A. Zelikin, S. Kargov, V. Izumrudov. Macromol. Chem. Phys. 202, 1368, (2001)

8. O. Davydova, A. Zelikin, S. Kargov, V. Izumrudov. Macromol. Chem. Phys. 203, 837, (2002).

9. H. Ríos, G. Collío, M. Leal, M. Urzúa, V. Vargas. J. Macromol. Sci. Part B. Phys. 52, 841, (2013).

10. H. Ríos, J. González-Navarrete, V. Vargas, M. Urzúa. Colloids Surface B. 384, 262, (2011).

11. R. Bazito, F. Cassio, F. Quina. Macromolecular Symp. 229, 197 (2005)

12. H. Vink. J.Chem.Soc. Faraday Trans. 1. 77, 2439, (1981)

13. R. Barraza, R. Martínez. Bol. Soc. Chil. Quím., 45, 563, (2000). 